\title{
Pretemporal craniotomy
}

\section{Craniotomia pré temporal}

\author{
Feres Chaddad-Neto 1,2,3, Hugo Leonardo Dória-Netto ${ }^{2,3}$, José Maria Campos-Filho2,3,
} Mateus Reghin-Neto ${ }^{2,3}$, Evandro Oliveira ${ }^{4}$

\begin{abstract}
This paper aims to describe the performance of the pretemporal craniotomy performed didactically from 2002 to 2012 in eighty patients. It is therefore a fundamentally descriptive text, organized in the sequence of the main stages in which such a craniotomy is performed, and describing in detail the technique with which this group of evolutionarily authors came to accomplish the task.
\end{abstract}

Keywords: neurosurgery, craniotomy, microsurgery.

\section{RESUMO}

O presente artigo visa descrever de forma didática e prática a realização da craniotomia pré-temporal aplicada em oitenta pacientes de 2002 a 2012. Trata-se, portanto, de um texto fundamentalmente descritivo, dividido conforme as principais etapas da realização desta craniotomia, e que descreve com detalhes a técnica com que o presente grupo de autores evolutivamente veio a realizá-la.

Palavras-chave: neurocirurgia, craniotomia, microcirurgia.

The pterional access, popularized by Yasargil in 1976, is, to date, the most widely used surgical route in neurosurgery ${ }^{1,2}$. This access allows for transylvian and lateral subfrontal views. As well as a straight downward view of the anterolateral aspect of the basilar bifurcation ${ }^{3,4}$.

Drake introduced the subtemporal approach during the $1970 \mathrm{~s}^{5,6}$. The subtemporal approach offers a lateral view of the interpenducular fossa by retracting the temporal lobe superiorly.

The temporopolar approach was first described by Sano in $1980^{7}$. The subtemporal approach offers a lateral view of the interpenducular fossa by retracting the temporal lobe superiorly. The temporopolar approach consists in pulling back the temporal pole, creating and enlarging an anterolateral view of the interpeduncular fossa.

The addition of transylvian and subfrontal views to the subtemporal and temporopolar views is of great importance when a neurosurgeon needs to expose the interpeduncular cistern region or the entire temporal lobe, including the medial portion. By microsurgical dissection of the Sylvian fissure, third cranial nerve, crural and ambiens we are able to expose the entire temporal lobe. The pretemporal approach, described by de Oliveira, combines the advantages of all these approaches in one craniotomy $y^{8-10}$. This approach exposes the entire temporal lobe in order to offer the transylvian and lateral subfrontal views, from the pterional craniotomy, along with the subtemporal and temporopolar views to access the interpeduncular fossa.

Our team has performed eighty pretemporal craniotomies from 2002 to 2012 at a private medical service. These were done to deal with forty six tumors, eighteen aneurysms, twelve arteriovenous malformations, two cavernous hemangioma and two dural arteriovenous fistula.

\section{DESCRIPTION OF PRETEMPORAL CRANIOTOMY}

\section{Positioning}

The patient should be placed supine, with the shoulder at the edge of the surgical table in a neutral position, and head and neck remain suspended after removal of the head support. The head should be secured by a three-pin skull fixation device (Mayfield or Sugita model), and must be maintained above the level of the right atrium to facilitate venous

\footnotetext{
${ }_{1}^{1}$ Professor of Neurosurgery, Departmento de Neurologia, Universidade Federal de São Paulo (UNIFESP), Sao Paulo SP, Brazil;

${ }^{2}$ Neurosurgeon, Instituto de Ciências Neurológicas, Sao Paulo SP, Brazil;

${ }^{3}$ Neurosurgeon, Hospital Beneficência Portuguesa de São Paulo, Sao Paulo SP, Brazil;

${ }^{4}$ Professor and Head of Neurosurgery, Department of Neurology, Faculty of Medical Sciences, Universidade Estadual de Campinas (UNICAMP), Campinas SP, Brazil. Coordinator of the Microneurosurgery Laboratory, Instituto de Ciências Neurológicas (ICNE), Hospital Beneficência Portuguesa, Sao Paulo SP, Brazil. Correspondence: José Maria de Campos Filho; Praça Amadeu Amaral 27 / $5^{\circ}$ andar / Bela Vista; 01327-010 São Paulo SP - Brasil; E-mail: icne@uol.com.br Conflict of interest: There is no conflict of interest to declare.

Received 31 March 2013; Received in final form 28 June 2013; Accepted 05 July 2013.
} 
return. In order to avoid the head holder position to hinder the surgeon's procedure, the ipsilateral pin of the operative field should be set on the mastoid region, while the two contralateral pins should be on the contralateral superior temporal line, above the temporal muscle, that should not be transfixed. The pin corresponding to the ipsilateral mastoid and the most anterior pin, corresponding to the contralateral superior temporal line, must be positioned in parallel to prevent any head movement, especially during subsequent traction of cranial wraps performed with the aid of fish-hooks.

Head positioning comprises a sequence of four movements for: lifting, deflection, rotation and torsion. In the lifting stage, the region to be operated is positioned at a level above the right atrium; deflection and rotation depend on the condition being operated; and in torsion, the angle formed by the head, neck and shoulder should increase, so as to offer the surgeon a closer lateral position with respect to the surgical area, in order to become parallel to the Sylvian fissure.

Bottom line is that care must be taken so that the jugular veins remain compression-free throughout surgery, to prevent delay of venous emptying, brain swelling, and increased bleeding in the operating field.

\section{Trichotomy}

Following induction of general anesthesia and proper catheterization, patient's hair should be combed with a brush used for hand scrubbing that has been soaked in detergent solution (chlorhexidine or polyvinylpyrrolidone), so as to facilitate shaving, that should be performed up to $2 \mathrm{~cm}$ from the region of the surgical incision. Shaving just prior to surgery allows for better fixation of fields, reduction of infection risks, and better fixation of the bandage after surgery. Once the area has been shaved, it is treated with ether-soaked gauze to remove the scalp fat, facilitate fixation of fields and the marking of the incision area with methylene blue. Shaving can also be performed following just the incision line, in a width of about $2 \mathrm{~cm}$.

\section{Marking, antisepsis and scalp incision}

After positioning and trichotomy, the skin incision is marked, so that its two ends form an imaginary straight line that adequately simulates the separation of the skin flap and the resulting bone exposure. Marking should be accurate, starting at the superior rim of the zygomatic arch anterior to the tragus, and extending to the hemi pupillary contralateral line in the frontal region, respecting the hairline whenever possible. Sometimes it is necessary to extend the incision up to the contralateral superior temporal line. The marked area anterior to the tragus should not be too anterior, so as to prevent sectioning the superficial temporal artery and the frontal branch of the facial nerve located anteriorly to that artery (Figure 1A and 1B).
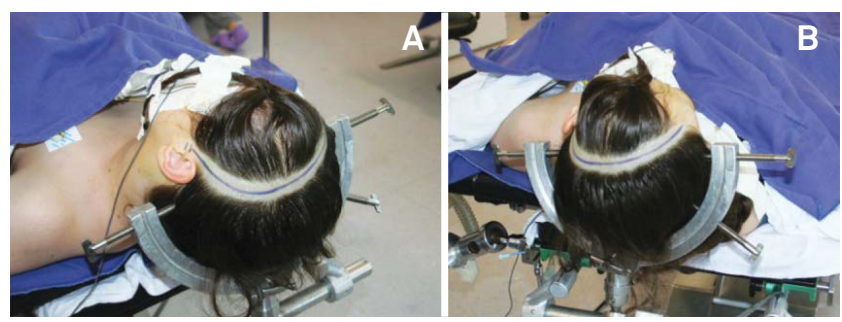

Figure 1. (A): The skin incision starts at the superior rim of the zygomatic arch anterior to the tragus. (B): The skin incision extends to the hemi pupillary contralateral line in the frontal region.

At this moment the arch of the surgical table that will serve as support for the tractions of the cutaneous, muscular and facial flaps must be properly positioned at a height that does not cause simultaneous compression of the eyeball. Ocular compression can cause blindness by thrombosis of the central retinal vein and thrombosis of the central retinal artery, especially in patients with shallow orbits, which is more common among yellow race peoples. A measuring device for determining the central venous pressure, positioned between the nasion and the arch of the surgical table allows for the angle of future tractions, in relation to the surface of the eyeball, to be estimated by triangulation.

Antisepsis should be achieved with povidone and benzoin should be subsequently applied to promote better adhesion in the region.

Scalp incision should be performed with a scalpel, and the use of bipolar coagulation helps to avoid bleeding of scalp arteries. The placement of wet gauze and later traction of the scalp flap can spare the use of haemostatic clips and specific staples for this purpose.

\section{Interfacial dissection, section and displacement of the temporalis muscle}

The interfacial dissection of the temporalis muscle, as originally described by Yasargil ${ }^{1,2}$, is specifically intended to preserve the frontotemporal branch of the facial nerve and reduce postoperative cosmetic changes resulting from the surgical wound.

The temporalis muscle is composed of two parts: an outer part which originates in the superior temporal line and inserts onto the coronoid process of the jawbone; and a deeper part that has its origin along the surface of the temporal squama and inserts onto the temporal crest of the jawbone. The temporalis muscle is covered by a superficial fascia which, in turn, consists of two layers (the superficial and deep layers). These are separated in their anterior portion by a pad of adipose tissue, and by a deep fascia that is more attached to the skull and protects, both, its vasculature (anterior, intermediate and posterior deep temporal arteries, branches of the maxillary artery) and its innervations (temporal branches of the mandible branches of the trigeminal nerve). 
Dissection of the superficial fascia should be performed vertically, starting from the superior temporal line, from 1.5 to $2 \mathrm{~cm}$ from the superior rim of the orbit to the posterior root of the zygomatic arch, with the aid of a cold \#13 scalpel and Metzenbaum scissors. The removal of the surface layer of the superficial temporal fascia and its underlying fat pad with the use of a hook placed at its center point facilitates completion of the dissection, whose basal layer is hindered by the presence of temporal nerves and vessels. With the most basal removal of the surface layer and the fat pad, good visualization of the deep muscular portion is achieved.

The dissection and detachment of the temporalis muscle are then performed in two stages. Initially we use the monopolar electrosurgery pencil (in the coagulation mode, and not for cutting, in order to avoid much bleeding) for the transversal section of the upper portion of the temporal muscle. We use the scalpel in a parallel position and about $1.5 \mathrm{~cm}$ inferior to the superior temporal line, moving from anterior to posterior, and leaving a top flap of temporal muscle attached to the skull surface for later suture of the inferior part that will be detached and that, during closure, will mainly cover the anterior burr hole. The second stage consists of performing the detachment of the deep muscular fascia of the skull, a procedure that should be carried out with the aid of a Cushing's elevator. The detachment of the deep temporal fascia is facilitated by starting at its posterior superior portion and using horizontal movements made with the tip of the elevator. After completing the detachment, the temporal muscles must be moved away, towards the posterior inferior section, with the aid of three hooks.

We need to expose and dislocate the zygomatic arch when a parallel microscopic view of the middle cranial base floor is needed, or in the case of patients with a thick temporal muscle. The edge of the inferolateral temporal lobe corresponds externally to the upper edge of the zygomatic arch. From the lower dislocation of the zygomatic arch, it is possible to inferiorly move the temporal muscle facilitating optimal exposure of the floor of the middle fossa. Using the number 1 Penfield dissector, we can move the muscle and connective tissue adherent to the zygomatic arch. The osteotomy is performed to the zygomatic arch anteriorly at the junction of the zygomatic arch with the frontozygomatic process of the frontal bone and posteriorly previously to the temporomandibular joint. The insertion of the masseter muscle in the lower portion of the zygomatic arch is preserved, this is then dislocated inferiorly.

\section{Craniotomy}

The main goal of pretemporal approach is to provide a basal and wide exposure of the temporal lobe and Sylvian fissure. We must expose the inferior frontal gyrus and a portion of the middle frontal gyrus, and the superior, middle and inferior temporal gyrus allowing for access through the transylvian, lateral subfrontal, temporopolar and subtemporal views.
The pretemporal craniotomy should be performed starting from three points of trepanation. The first trepanation must be set between the superior temporal line and the frontozygomatic suture of the external orbital process; the second trepanation is performed on the most posterior extension of the superior temporal line and the third one should be made on the most inferior portion of the squamous part of the temporal bone. Since the lesser wing of the sphenoid bone is situated internally between the first and third trepanations, and this bone rim will be properly removed through drilling, the third trepanation should not be performed very close to the base to facilitate the later cutting of the bone between these two trepanations. In cases of a prominent sphenoid wing, the osteotomy of that segment should be complemented with the use of drilling, as described below. After the trepanations, the dura must be properly detached from the internal bone surface with the aid of dissectors suitable for this purpose.

Craniotomy may be performed with a Gigli saw or a craniotome, always making the cut at the level of the outer edge of each trepanation. In the cases where the lesser sphenoid wing is so prominent, to the point that it prevents the complete osteotomy between the first and third trepanations, the bone flap should be removed only after performing a superficial drilling of approximately $1 \mathrm{~cm}$ of the lateral surface of the greater wing of the sphenoid bone, as mentioned above.

Following proper hemostasis with bipolar electrosurgical forceps, always set at low power to avoid further retractions, the dura must be anchored with 4.0 nylon or prolene through perforations made along the bone ridge, seeking, thereby, to prevent the formation of extradural blood collections, both trans and postsurgery.

\section{Basal drilling}

The purpose of drilling the lesser wing of the sphenoid bone, the orbital roof and what remains of the temporal squama is to achieve bone flattening to facilitate the basal access with minimal brain retraction, which will be further optimized with cisternal opening and the aspiration of cerebrospinal fluid.

Firstly, the dura is detached from the orbital roof and from what remains of the lesser wing of the sphenoid with the use of appropriate dissectors, in order to attain better exposure of these bone surfaces and in preparation for the eventual placement of the spatula fixed to the orthostatic retractor that, if necessary, can also be used as a protective element for the dural surface. Drilling should be initiated on the outermost section of the orbital roof with the use of a cylindrical or round drill, seeking the removal of its bony prominences. Then, the base of the remaining temporal squama must be drilled so as to leave the lesser wing of the sphenoid projected between the orbital roof and the previously drilled temporal base. The lesser wing of the sphenoid bone should 
then be drilled following repositioning of the spatula on the dural impression of the sphenoid, until we obtain visibility of the dural cuff that contains the meningo-orbital artery located at the superolateral level of the superior orbital fissure. After its identification, the meningo-orbital artery should be isolated, coagulated and sectioned with a cold blade scalpel \#11, with its cutting side facing the lesser wing of the sphenoid, and the concomitant posterior traction of the dura.

Drilling of the entire lateral surface of the larger wing of the sphenoid should be performed so as to expose the entire temporal pole, to achieve the temporopolar view. Drilling of the middle fossa floor to the foramen spinosum is done to expose the entire bottom surface of the temporal lobe to achieve the subtemporal route. Therefore, the drilling of the squamous temporal bone and greater wing of sphenoid bone should be performed until the floor of the middle cranial fossa is completely exposed, with complete release of the anterior and basal surfaces of the temporal lobe, this constitutes the difference between pretemporal and pterional craniotomies (Figure 2).

The most delicate drillings must be made with the use of match-shaped drills or diamond drills, whenever possible. When the dural resistance is intense, a small incision of the dura at the level of the projection of the lateral fissure can be made, in order to enable cerebrospinal fluid drainage, and subsequent dural relaxation.

It is important to point out that the dural detachment should not exceed the limits of the drilling, thus preventing creation of dead extradural spaces. Dural anchoring should be complemented along the temporal bone ridge after drilling has been completed.

\section{Draping the operative field over the bony ridge}

After positioning and arrangement of rectangular cotton blocks on the free bony ridge, blue drapes are placed on the pieces of cotton, aiming to cover the superficial cranial wraps and minimize the further reflection of light from the surgical microscope.

\section{Opening the dura mater and brain exposure}

Opening of the dura mater should be performed in such a way that, when folded back, the external dural surface adapts itself to the bone surface without forming wrinkles or folds that might obstruct the microneurosurgical field.

At the end of the dural opening, the shape should be of that of a large $\mathrm{S}$ so as to circumvent the temporal lobe, with the concave portion toward the roof top free of orbit and the bottom toward the edge and bottom of the posterior craniotomy.

The dural incision should be initiated near the second trepanation; so, it should be initiated at the level of the most frontoparietal aspect of the dural exposure, using a scalpel blade \#11, and continued in the frontal superior direction, at this point with the use of Metzenbaum scissors. It then

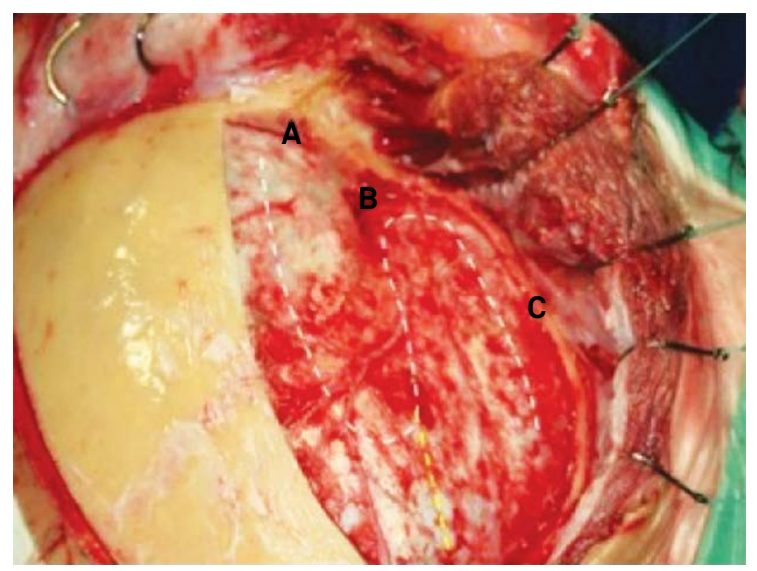

Figure 2. Pretemporal craniotomy, the greater and lesser wing of the sphenoid, the squamosal part of the temporal bone and the orbital roof are drilled out. The line shows the $\mathrm{S}$ shape dural opening. A: orbital roof, B: sphenoid wing region, C: middle fossa floor.

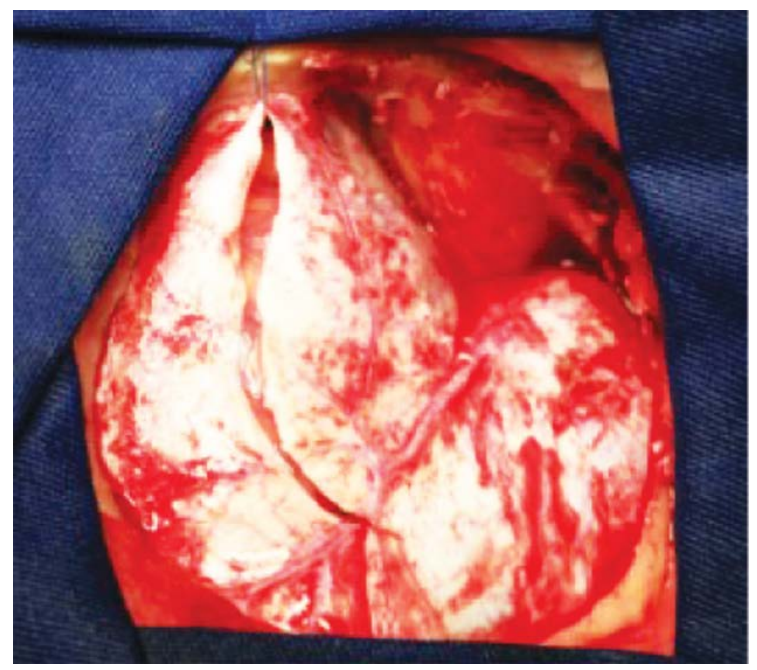

Figure 3. Initially the dura is opened at the frontal region.

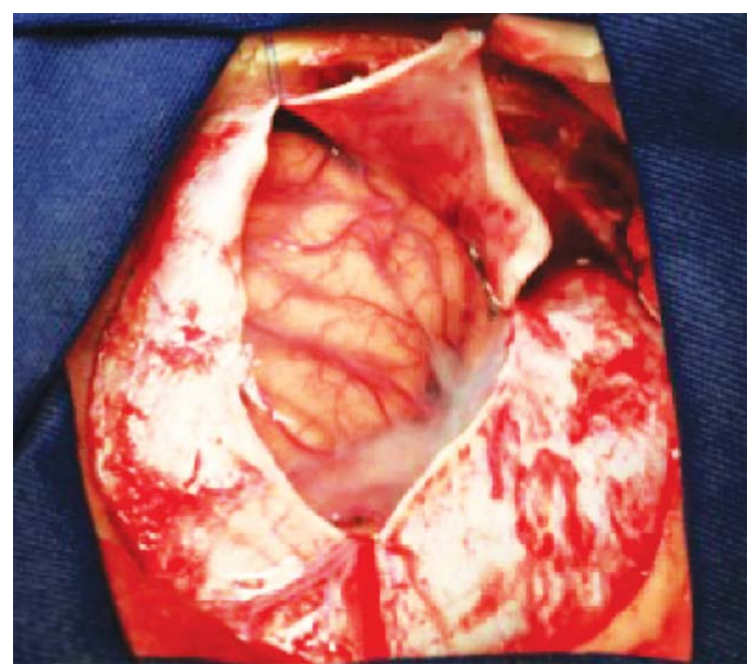

Figure 4. The dura is opened through the sylvian fissure toward the superior orbital fissure. The dura is reflected on the orbital roof without causing folds or ridges. 
follows toward the Sylvian fissure, and then upward towards the superior orbital fissure and, finally, turns posteriorly, outlining the middle cranial fossa so as to fully expose the temporal lobe. The flap should be anchored with 4.0 nylon or prolene thread and pulled back in order to lift up the dural edges (Figures 3, 4 and 5).

With this form of dural opening the front flap can be reflected and pulled on the orbital roof without causing major folds or ridges of the dura mater, and the temporal flap can be reflected in the same way over the lower and posterior edge of the craniotomy, thus exposing the lateral fissure and the entire temporal lobe (Figure 6).

\section{Opening of the Sylvian fissure}

The Sylvian fissure is composed of a superficial and a deep part. The superficial part presents a stem and three branches; the stem extends medially from the semilunar gyrus of the uncus, between the basal surface of the frontal

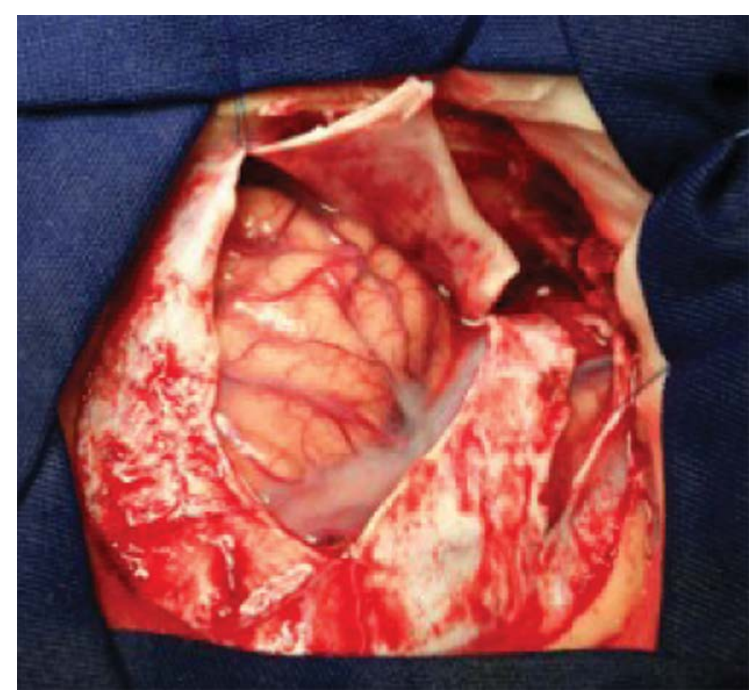

Figure 5. Finally the temporal lobe is outlined after the $\mathrm{S}$ shape dural open.

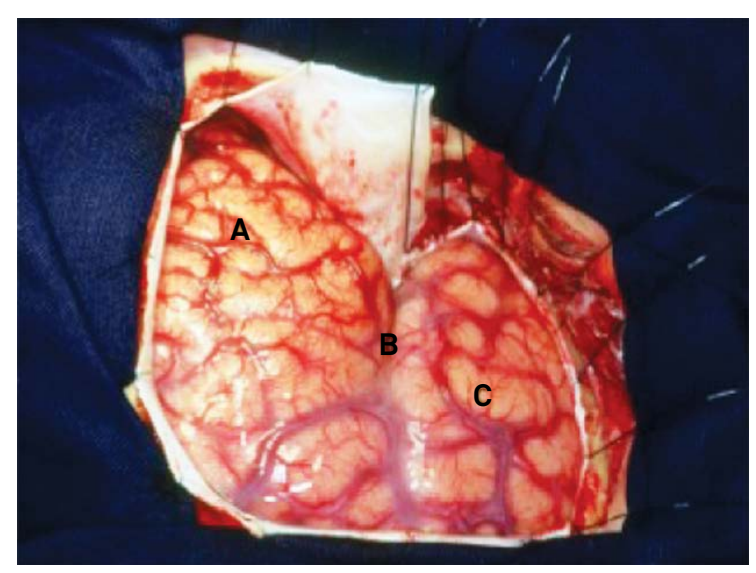

Figure 6. After the dural opening, the lateral fissure and the entire temporal lobe are exposed. A: frontal lobe, B: lateral fissure, C: temporal lobe. lobe and the pole of the temporal lobe to the lateral end of the sphenoid ridge, where the stem divides itself into anterior horizontal, anterior ascending, and posterior branches. The deep part is divided in an anterior part, the sphenoidal compartment, and a posterior part, the operculoinsular compartment. The sphenoidal compartment arises in the region of the limen insulae, at the lateral margin of the anterior perforated substance. The sphenoidal compartment is a narrow space posterior to the sphenoid ridge, between the frontal and the temporal lobes, that communicates medially with the carotid cistern. The operculoinsular compartment is formed by two narrow clefts, the opercula cleft between the opposing lips of the frontoparietal and the temporal opercula and the insular cleft. The insular cleft has a superior limb located between the insula and the frontoparietal opercula and an inferior limb between the insula and the temporal operculum. The opercular cleft is composed of the frontal and parietal opercula superiorly and the temporal operculum inferiorly.

When the lips of the Sylvian fissure are widely separated we can see the insula. The insula connects the temporal lobe to the posterior orbital gyrus via the limen insulae. The limen insulae serves as a threshold between the carotid cistern (also called Sylvian vallecula) medially and the Sylvian fissure laterally. From microsurgical and radiologic viewpoints, the insula represents the external covering of a mass comprised of the extreme, external, and internal capsules, claustrum, basal ganglia, and thalamus ${ }^{11-14}$.

The pretemporal approach proceeds with the opening of the Sylvian fissure and the basal cisterns. We usually open the basal cisterns before the Sylvian fissure to drain the cerebrospinal fluid, thus relaxing the brain, it makes the split of the Sylvian fissure easier. This splitting usually begins at the level of the pars triangularis, where the space between the frontal and the temporal lobes is wider.

The superficial part of the Sylvian fissure is opened using a scapel blade number 11 and microscissors. The dissection is usually done on the frontal side of the superficial Sylvian vein, leaving its superficial part to the temporal side. The superficial Sylvian vein commonly courses laterally toward the tip of the temporal lobe to empty into the sphenoparietal sinus. Sometimes the superficial Sylvian vein drains predominantly toward the frontal Sylvian vein, then into the superior sagittal sinus. Because of this, the splitting of the superficial part of the Sylvian fissure is performed laterally to the superficial Sylvian vein.

When the superficial part of the frontal and the temporal lobes are extremely adherent it is advisable to deepen the dissection into the operculoinsular compartment of the Sylvian fissure to identify the branches of the middle cerebral artery. After that, we dig a tunnel along the deep part of the Sylvian fissure coming back to the surface splitting the superficial part of the Sylvian fissure. 


\section{The basal cisterns}

The pretemporal craniotomy enables the surgeon to reach the olfactory cistern, the carotid cistern, the chiasmatic cistern, the sphenoid compartment of the Sylvian fissure, the lamina terminalis cistern, the interpeduncular cistern, the ambient cistern and the crural cistern, that can be reached after the removal of the anteromedial segment of the uncus (Figures 7, 8 and 9).

In order to achieve access to the interpeduncular cistern, the bridging veins draining the temporal pole to the sphenoparietal sinus and the veins from the orbital surface of the frontal lobe to the sphenoparietal and cavernous sinuses are sacrificed.

The arachnoid that binds the uncus to the oculomotor nerve and to the tentorial edge is opened, in order to achieve a nice mobility of the temporal lobe. After the cisternal opening the temporal pole can be elevated superiorly

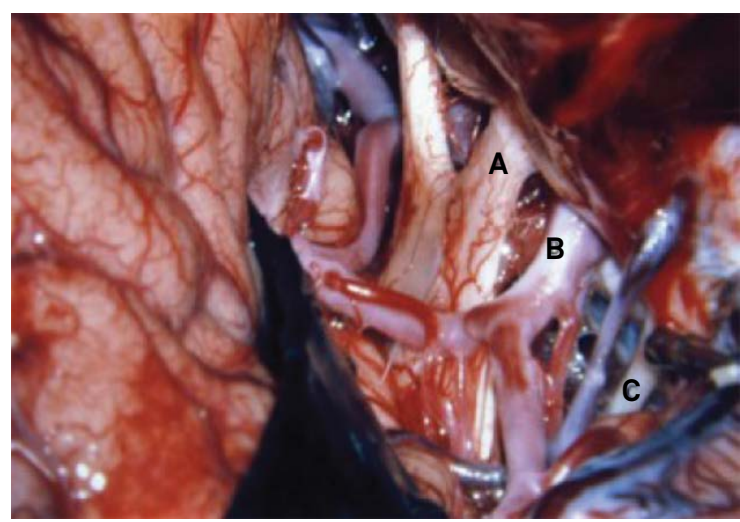

Figure 7. The interpeduncular cistern can be accessed between the internal carotid artery and the optic nerve, between the internal carotid artery and the third nerve and retracting or opening the tentorium cerebelli laterally of the third nerve. A: optic nerve, B: carotid artery, C: oculomotor nerve.

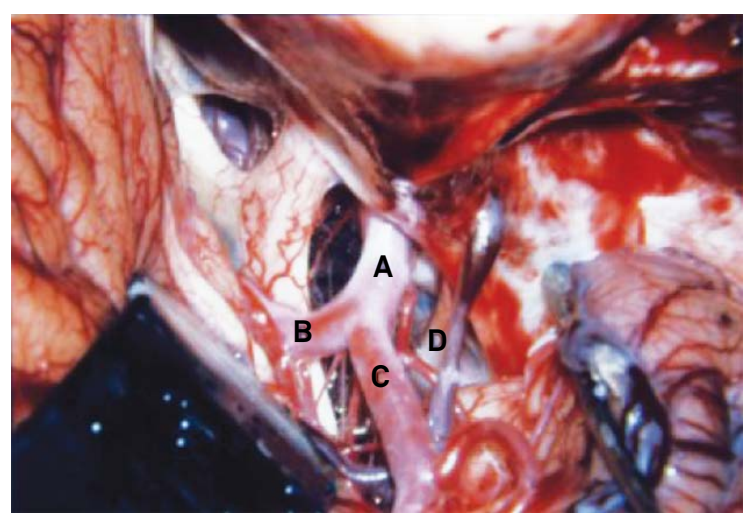

Figure 8. The pretemporal approach allows a large exposure of the basal cisterns and the visualization of the carotid artery and its bifurcation, the optic nerve, the third nerve. A: carotid artery, B: anterior cerebral artery, C: middle cerebral artery, D: oculomotor nerve. through the subtemporal route and posteriorly through the temporopolar route (Figures 10 and 11).

The steps followed in performing pretemporal craniotomies have been facilitated dealing with these eighty cases from 2002 to 2012.

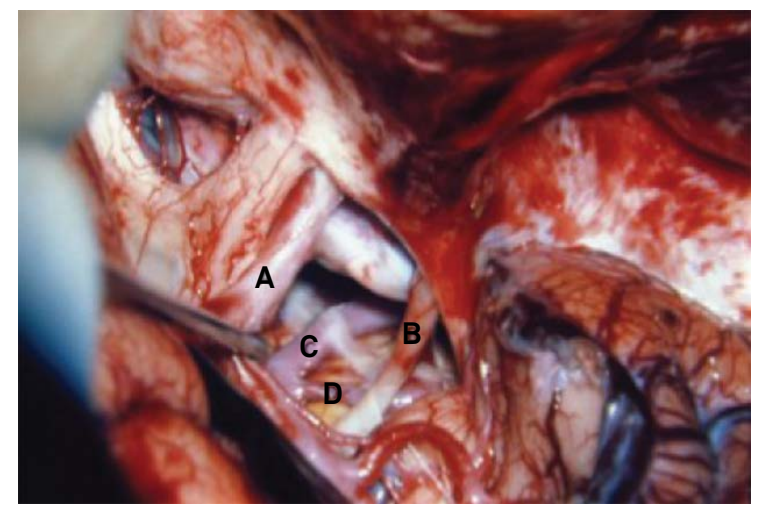

Figure 9. The antero-lateral route shows the interpeduncular cistern through the space between the carotid artery and the third nerve, exposing the basilar artery. A: carotid artery, B: oculomotor nerve, C: basilar artery, D: superior cerebellar artery.

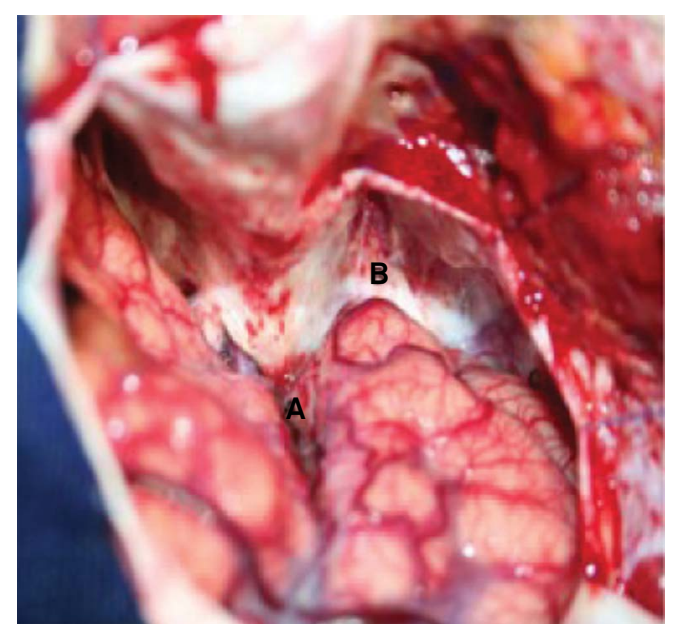

Figure 10. Pretemporal craniotomy exposes trans sylvian (A), temporopolar (B) and subtemporal (C) approaches.

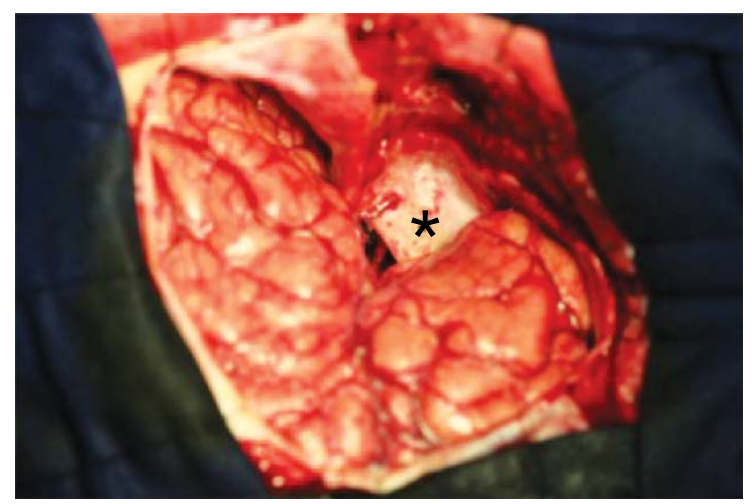

Figure 11. Temporopolar view $(*)$. 
1. Yasargil MG, Antic J, Laciga R, et al. Microsurgical pterional approach to aneurysms of the basilar bifurcation. Surg Neurol 1976;6:83-91.

2. Yasargil MG. Basilar artery bifurcation aneurysms. In: Yasargil Mg (Ed). Microneurosurgery, vol 2 Stuttgart, Geor Thieme Verlag, 1984:232-246

3. Chaddad-Neto F, Campos Filho JM, Doria Netto HL, Faria MH, Ribas GC, De Oliveira E. The pterional craniotomy: tips and tricks. Arq Neuropsiquiatr 2012;70:727-732.

4. Chaddad-Neto F, Ribas GC, De Oliveira E. The pterional craniotomy step by step. Arq Neuropsiquiatr 2007;65:101-106.

5. Drake CG. The surgical treatment of aneurysms of basilar artery. $J$ Neurosurg 1968;29:436-446.

6. Drake CG. The treatment of aneurysms of the posterior circulation. Clin Neurosurg 1979;26:96-144.

7. Sano K. Temporo-polar approach to aneurysms of the basilar artery at and around the distal bifurcation: Technical note. Neurol Res 1980;2:361-367.
8. De Oliveira E, Siqueira M, Tedeschi H, Peace DA: Surgical approaches for aneurysms of the basilar artery bifurcation. In: Matsushima T (Ed). Surgical anatomy for microneurosurgery VI: cerebral aneurysms and skull base lesions. Fukuoka City, Japan, Sci Med Publications, 1993:34-42.

9. De Oliveira E, Tedeschi H, Siqueira Mg, Peace DA. The pretemporal approach to the interpeduncular and petroclival regions. Acta Neurochir (Wien) 1995;136:204-211.

10. Tedeschi H, De Oliveira E, Wen HT. Pretemporal approach to basilar bifurcation aneurysms. Techn Neurosurg 2000;6:191-199.

11. Ribas GC, Ribas EC, Rodrigues CJ. The anterior Sylvian point and the suprasylvian operculum. Neurosurg Focus 2005;18:E1-E6.

12. Yasargil MG. Microneurosurgery. Stuttgart: Georg Thieme, 1984.

13. Yasargil MG, Krisht AF, Türe U, Al-Mefty O, Yasargil DCH. Microsurgery of insular gliomas: part I. Surgical anatomy of the Sylvian cistern. Contemp Neurosurg 2002;24:1-8.

14. Yasargil MG, Krisht AF, Türe U,Al-Mefty O, Yasargil DCH. Microsurgery of insular gliomas: part II. Opening of the sylvian fissure. Contemp Neurosurg 2002;24:1-5. 